\title{
Photo-Realistic Tissue Reflectance Modelling for Minimally Invasive Surgical Simulation
}

\author{
Mohamed A. ElHelw ${ }^{1}$, Stella Atkins ${ }^{2}$, Marios Nicolaou ${ }^{1}$, \\ Adrian Chung ${ }^{1}$, and Guang-Zhong Yang ${ }^{1}$ \\ ${ }^{1}$ Department of Computing, Imperial College London, UK \\ \{mohammed.elhelw, m.nicolaou, a.chung, g.z.yang\} aimperial.ac.uk \\ ${ }^{2}$ School of Computer Science, Simon Fraser University, Canada \\ stella@cs.sfu.ca
}

\begin{abstract}
Computer-based simulation is an important tool for surgical skills training and assessment. In general, the degree of realism experienced by the trainees is determined by the visual and biomechanical fidelity of the simulator. In minimally invasive surgery, specular reflections provide an important visual cue for tissue deformation, depth and orientation. This paper describes a novel image-based lighting technique that is particularly suitable for modeling mucous-covered tissue surfaces. We describe how noise functions can be used to control the shape of the specular highlights, and how texture noise is generated and encoded in image-based structure at a pre-processing stage. The proposed technique can be implemented at run-time by using the graphics processor to efficiently attain pixel-level control and photo-realism. The practical value of the technique is assessed with detailed visual scoring and cross comparison experiments by two groups of observers.
\end{abstract}

\section{Introduction}

With the maturity of Virtual Reality (VR) and Augmented Reality (AR), there is now a wide spread use of the techniques in Minimally Invasive Surgery (MIS). Intraoperatively, AR enables navigation in image-guided surgery where the surgeon's view of the operating field is augmented by the addition of computer-generated data. $\mathrm{VR}$, on the other hand, is increasingly being used in a mixed reality environment for surgical simulation. Pre-operatively, VR simulators are used to train practitioners in basic surgical tasks as well as complete interventions. They have been found to be particularly useful for MIS procedures because of the complexity of instrument controls, restricted vision and mobility, difficult hand-eye co-ordination, and the lack of tactile perception require a high degree of operator dexterity [1]. MIS simulations provide an economical and time saving solution for acquiring, as well as assessing, basic surgical skills [2]. They offer the opportunity for safe, repeated practice and objective measurement of surgical performance.

One of the key issues to be addressed in surgical simulation is visual realism and mechanical fidelity. The creation of realistic lighting can greatly affect visual realism and hence the overall quality of the simulation. Traditional methods have found limited use in advanced surgical training, particularly for those involving patient 
specific models. To this end, a number of physically-based approaches for computing diffuse and specular light components have been proposed. Diffuse reflection modelling considers diffuse reflection as the result of light entering the surface, scattering, interacting with its material and then exiting in random directions [3]. Relative contributions of surface reflection and sub-surface scattering and transmission are computed, therefore allowing for simulating layered materials and biological tissues. For specular reflection, a number of micro-facet models are used for simulating specular surfaces [4], [5]. These models consider rough surface topology with perfect micro-reflectors accounting for the spectral composition of specular highlights. They are further extended to handle wide-ranging surface types [6] and anisotropic distributions with multiple scattering. Despite the visual realism achieved, physically-based reflection modelling is not particularly suitable for interactive surgical simulation because of its inherent high computational costs.

Existing research has shown that specular highlights constitute a major visual cue for gauging tissue deformation, depth and orientation during MIS procedures [7]. Reproducing the same effect in a simulated environment is difficult due to the nature of tissue, which is comprised of multiple layers covered with translucent mucous. The purpose of this paper is to present a novel photorealistic lighting method for simulating the appearance of specular highlights reflected from the internal lumen. The method is based on using noise functions to modulate the shape of specular highlights and imitate the effects of composite multi-layer tissue types on reflected lighting. We will describe how texture noise is generated and encoded in image-based structures at a preprocessing stage and show how this information is used during run-time by the graphics processor to efficiently attain pixel-level control and achieve photorealistic tissue appearance. The visual realism achieved by the technique is assessed by two groups of observers with detailed visual scoring and cross comparison.

\section{Method}

\subsection{Surface Reflectance Modeling}

The use of computer-generated noise is a well-established method for representing natural scenes such as terrain topography, clouds, vegetation distributions, fur/hair, as well as natural effects such as fire, fluid flow and erosion patterns [8-11]. In this study, the modeling of specular highlights is achieved by using 2D Perlin noise [12], which first creates a noise image followed by converting it to a reflectance map. For each point in the noise image, the associated tangent vectors to the surface are defined, and each pixel in a reflectance map encodes the direction that the corresponding point is facing by using three channels of information that are conveniently mapped to a standard Red-Green-Blue (RGB) image.

Since a reflectance map is essentially an RGB image, per-triangle noise information is extracted during run-time by means of texture mapping for calculating the specular highlights. The extracted noise vectors are defined in image coordinate space, so they have to be transformed into a coordinate that is local to the triangle being processed. The basis vectors of the triangle local coordinate system, known as the surface-local coordinate space, can be defined by using the surface tangent $(T)$, bitangent $(B)$, and the normal $(N)$, where the first two vectors can be computed from the 
partial derivatives of the object-space coordinates of the triangle in terms of its texture coordinates [13], i.e.,

$$
T=(\partial x / \partial u, \partial y / \partial u, \partial z / \partial u)=\left(-T_{0},-T_{1},-T_{2}\right)
$$

where

$$
\begin{aligned}
& T_{0}=\left[\left(v_{1}-v_{0}\right)\left(x_{2}-x_{0}\right)-\left(x_{1}-x_{0}\right)\left(v_{2}-v_{0}\right)\right] /\left[\left(u_{1}-u_{0}\right)\left(v_{2}-v_{0}\right)-\left(v_{1}-v_{0}\right)\left(u_{2}-u_{0}\right)\right] \\
& T_{1}=\left[\left(v_{1}-v_{0}\right)\left(y_{2}-y_{0}\right)-\left(y_{1}-y_{0}\right)\left(v_{2}-v_{0}\right)\right] /\left[\left(u_{1}-u_{0}\right)\left(v_{2}-v_{0}\right)-\left(v_{1}-v_{0}\right)\left(u_{2}-u_{0}\right)\right] \\
& T_{2}=\left[\left(v_{1}-v_{0}\right)\left(z_{2}-z_{0}\right)-\left(z_{1}-z_{0}\right)\left(v_{2}-v_{0}\right)\right] /\left[\left(u_{1}-u_{0}\right)\left(v_{2}-v_{0}\right)-\left(v_{1}-v_{0}\right)\left(u_{2}-u_{0}\right)\right]
\end{aligned}
$$

and

$$
B=(\partial x / \partial v, \partial y / \partial v, \partial z / \partial v)=\left(-B_{0},-B_{1},-B_{2}\right)
$$

where

$$
\begin{aligned}
& B_{0}=\left[\left(x_{1}-x_{0}\right)\left(u_{2}-u_{0}\right)-\left(u_{1}-u_{0}\right)\left(x_{2}-x_{0}\right)\right] /\left[\left(u_{1}-u_{0}\right)\left(v_{2}-v_{0}\right)-\left(v_{1}-v_{0}\right)\left(u_{2}-u_{0}\right)\right] \\
& B_{1}=\left[\left(y_{1}-y_{0}\right)\left(u_{2}-u_{0}\right)-\left(u_{1}-u_{0}\right)\left(y_{2}-y_{0}\right)\right] /\left[\left(u_{1}-u_{0}\right)\left(v_{2}-v_{0}\right)-\left(v_{1}-v_{0}\right)\left(u_{2}-u_{0}\right)\right] \\
& B_{2}=\left[\left(z_{1}-z_{0}\right)\left(u_{2}-u_{0}\right)-\left(u_{1}-u_{0}\right)\left(z_{2}-z_{0}\right)\right] /\left[\left(u_{1}-u_{0}\right)\left(v_{2}-v_{0}\right)-\left(v_{1}-v_{0}\right)\left(u_{2}-u_{0}\right)\right]
\end{aligned}
$$

In the above equations, $\left(x_{0}, y_{0}, z_{0}\right),\left(x_{1}, y_{1}, z_{1}\right),\left(x_{2}, y_{2}, z_{2}\right)$ and $\left(u_{0}, v_{0}\right),\left(u_{1}, v_{1}\right),\left(u_{2}, v_{2}\right)$ represent the object- and texture-space coordinates respectively for the prospective triangle. Subsequently, $(N)$ can be calculated from the cross product of $(T)$ and $(B)$ or alternatively the normal supplied by the original model can be used. By computing the basis vectors of the surface-local coordinate space, the GPU can then be used to efficiently transform the extracted image-space noise vectors to the surface-local space and carry out specular highlight computations.

In order to evaluate the effects of adding specular highlights on visual realism, subject-specific textures of 3D models were derived. Video bronchoscope images (Olympus BF Type; with field of view $120^{\circ}$ ) were registered with CT scans (Siemens Somaton Volume Zoom four-channel multi-detector) for five patients. This enabled the exact camera pose of the bronchoscope examinations to be identified. The surface details including texture and shading parameters are also extracted. The texture map is derived directly from the video bronchoscope images. The shading parameters are recovered by modeling the bidirectional reflectance distribution function (BRDF) $\rho$ of the visible surfaces by using a cubic curve parameterized on $\gamma$, the cosine of the angle between the viewing vector $(V)$ and surface normal $(N)$ [14]:

$$
\rho(V, N)=\sum_{0}^{3} c_{i} B_{i}^{3}(\gamma)
$$

where

$$
\gamma=\frac{V . N}{|V \| N|}, B_{i}^{n}(t)=\frac{n !}{i !(n-i) !}(1-t)^{(n-i)} t^{i}
$$

\subsection{Assessment of Visual Realism}

A user study was conducted to evaluate the effects of the proposed surface reflectance modeling for added visual realism. Participants from two subject groups were 
considered, where the first group consisted of 16 computing science students, and the second group consisted of 7 experienced bronchoscopists who had each performed between 150 and 2000 endoscopy procedures. All subjects had normal or corrected vision and were assumed to have good comprehension and analytical skills.

Static images on a computer monitor were presented to each subject for evaluation, one image at a time. The images show views of the bronchial tree from 8 poses from 5 different patients (estimated from camera parameters and used for generating synthetic views). Five images were created for each pose comprising five different categories ranging from least realistic to real. Category 1 uses the conventional shading algorithm that represents the lowest quality in the evaluation scale, whereas Category 5 is a real captured video bronchoscope image. Category 3 represents images rendered with subject-specific texture extracted with the BRDF method described above after 2D/3D registration. Category 4 is as category 3 but with specular highlights added by using the proposed technique to improve visual realism. For Category 2, low-resolution surface texture was used. The participants were first shown two examples of category 1 (most unrealistic) and category 5 (real) images displayed side-by-side for visual calibration. Then the subjects were presented with series of 15 images and asked to rank each image in terms of visual realism by following the Likert scale (1 to 5). A two alternative forced choice (2AFC) experiment was also conducted. In this experiment, the subjects viewed 23 side-byside image pairs from categories 3,4 and 5, always from different poses, and were asked to choose which of the two (left or right) was the most realistic. It is worth noting that during the experiments, no time limits were imposed and the images were displayed in a random order. The subjects were not told which the real images were, nor were they informed how the images were obtained.

\section{Results}

Figure 1 depicts the results obtained by implementing the described method in $\mathrm{Cg}$ [15] on NVIDIA FX graphics hardware compared with the conventional OpenGL multi-texturing approach. It can be seen that the method effectively avoids the problem of plastic-like surface appearance and provides realistic specular highlights. Furthermore, by varying the colour of the mucous layer and using different noise types, tissue appearance can be modified. The bottom row of Figure 1 further demonstrates the effect of different noise functions on the visual appearance of the rendered surface, where the frequency of the noise function is increased (left to right).

In Figure 2, the general appearance of the five category images used in the user study for assessing visual realism is illustrated. This demonstrates the increased visual realism that is achieved by using the proposed algorithm. As mentioned earlier, the last image category represents the real bronchoscope image.

Figure 3 summarizes the mean score for all the images of each category, averaged over all the participants for each group. It is seen that the overall score of all the subjects shows a steady increase in the scored realism using the proposed method for enhancing visual realism. It is also evident that the expert group is not significantly different to the naïve group in judging realism. This shows that results using naïve subjects to test for realism of tissue samples may transfer to training simulators for physicians. 

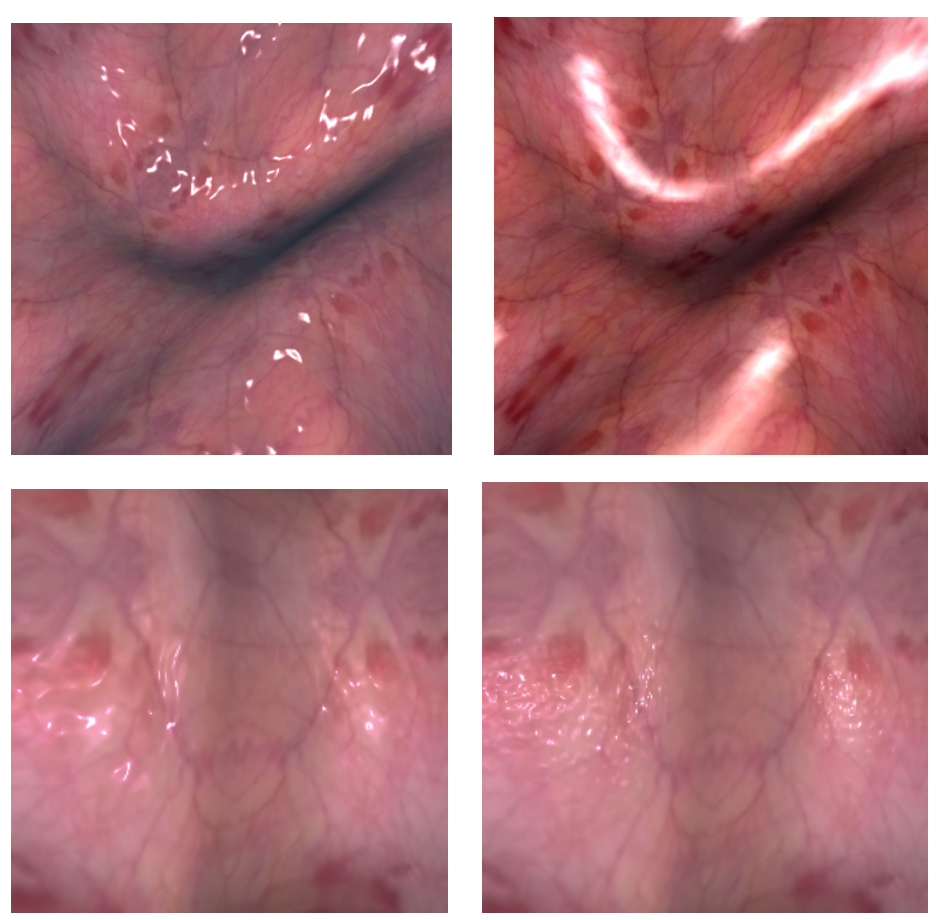

Fig. 1. (Top) Two views of the same surface rendered by using the proposed method (left) versus OpenGL multi-texturing (right). Notice the plastic-like surface and the hexagonal shape of the specular highlights rendered with the multi-texturing method (Bottom) By varying the frequency of the noise function, different specular appearance of the tissue can be simulated.

A more detailed statistical summary is provided in Table 1, which uses the combined realism scores from all the participants for images of Categories 3, 4 and 5 . By applying a paired two-tailed $t$-test to the data, synthetic images of Category 3 without specular highlights had a significantly lower score than the images with specular highlights added $(p=0.057)$ and also a significantly lower score than the real images $(p=0.026)$. It is also shown that there is no significant difference between synthetic images with highlights and real images $(p=0.780)$ which indicates that the Category 4 specular highlights can be perceived as close to photorealism.

For the 2AFC test mentioned above, it was found that when comparing a Category 3 image side-by-side with a Category 4 image, the Category 4 image was selected as the most realistic $68 \%$ of the time. Therefore missing specular highlights reduced the users' perception of reality. This result was confirmed when comparing Category 3 images with real (Category 5) images, where the real images were selected as being more realistic $73 \%$ of the time. However, when comparing Category 4 images with real images, the real images were selected as being more realistic only $48 \%$ of the time. This shows how the addition of noise-based specular highlights positively affected users' judgment of realism. 


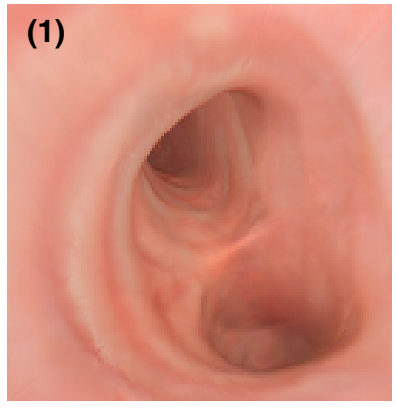

(4)

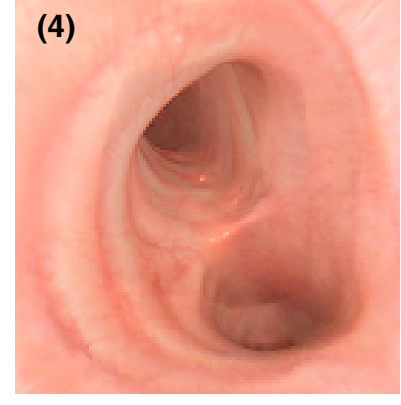

(2)

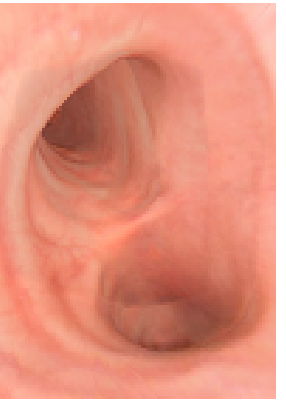

(3)

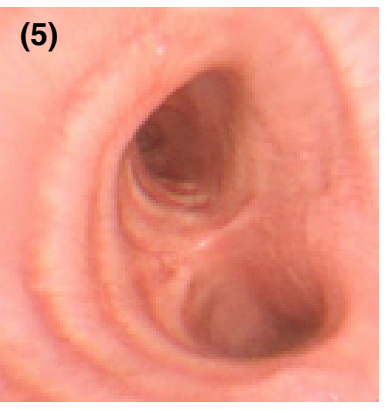

Fig. 2. The five different categories used as the stimuli for one pose are defined as follows (1) Unreal (2) BRDF-low resolution (3) BRDF (4) BRDF-Specular (5) Real bronchoscope image

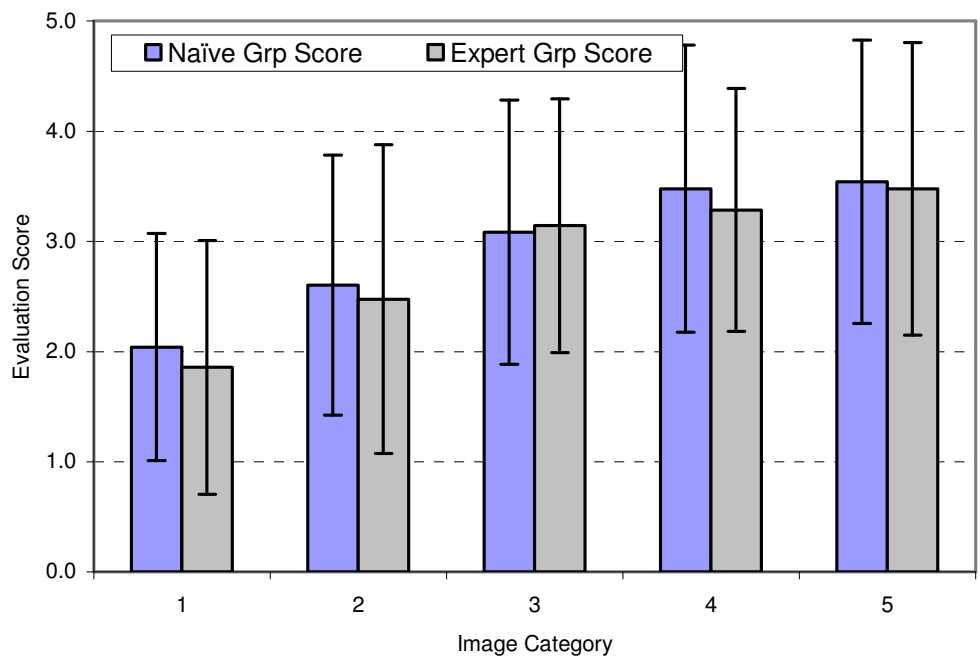

Fig. 3. Average score for all the images of each category, averaged over all the participants for each group. Error bars show one standard deviation 
Table 1. Details of the $t$-test with the scores of images from Categories 3, 4 and 5

\begin{tabular}{|c|c|c|c|c|c|c|}
\hline & \multicolumn{2}{|c|}{ Cat 3 Vs. Cat 4} & \multicolumn{2}{|c|}{ Cat 3 Vs. Cat 5} & \multicolumn{2}{|c|}{ Cat 4 Vs. Cat 5} \\
\hline & Cat3 & Cat4 & Cat3 & Cat5 & Cat4 & Cat5 \\
\hline Mean & 3.08 & 3.48 & 3.08 & 3.54 & 3.48 & 3.54 \\
\hline Variance & 1.44 & 1.70 & 1.44 & 1.66 & 1.70 & 1.66 \\
\hline $\begin{array}{l}\text { Hypothesized Mean } \\
\text { Diff }\end{array}$ & \multicolumn{2}{|c|}{0.00} & \multicolumn{2}{|c|}{0.00} & \multicolumn{2}{|c|}{0.00} \\
\hline$t$ Stat & \multicolumn{2}{|c|}{-1.94} & \multicolumn{2}{|c|}{-2.30} & \multicolumn{2}{|c|}{-0.28} \\
\hline$p(T<=t)$ two-tail & \multicolumn{2}{|c|}{0.057} & \multicolumn{2}{|c|}{0.026} & \multicolumn{2}{|c|}{0.78} \\
\hline$t$ Critical two-tail & \multicolumn{2}{|c|}{2.01} & \multicolumn{2}{|c|}{2.01} & \multicolumn{2}{|c|}{2.01} \\
\hline
\end{tabular}

\section{Conclusion}

In this paper, a novel photorealistic rendering method suitable for MIS simulation is described. The rendering method is based on using reflectance maps to model the effect of surface details and mucous layer on overall tissue appearance. For efficiency, reflectance map generation is achieved in pre-processing. During run-time, the graphics processor is used to allow for per-pixel control and rendering efficiency. The visual assessment results from the user study have shown that when combined with subject specific texture extraction through a BRDF model, the difference between images derived from the proposed method and real video bronchoscope is minimal. The results have also shown that the visual score derived had little difference between the naïve and expert groups, thus highlighting the potential value of the technique for both basic and advanced surgical skills training and assessment.

\section{References}

1. Bro-Nielsen, M.: Simulation Techniques for Minimally Invasive Surgery. Journal of Minimally Invasive Therapy \& Allied Technologies (1997) 106-110

2. Shah, J., Darzi, A.: Simulation and Skills Assessment. International Workshop on Medical Imaging and Augmented Reality (MIAR '01), Hong Kong (2001) 5-9

3. Hanrahan, P., Wolfgang, K.: Reflection from Layered Surfaces due to Subsurface Scattering. In Kajiya, J.T. (ed.): Computer Graphics (Proceedings of ACM SIGGRAPH '93 Conference), Vol. 27. Annual Conference Series, ACM Press, New York (1993) 165-174

4. Blinn, J.F.: Light Reflection Functions for Simulation of Clouds and Dusty Surfaces. In Bergeron, R.D. (ed.): Computer Graphics (Proceedings of ACM SIGGRAPH '82 Conference), Vol. 16. Annual Conference Series, ACM Press, New York (1982) 21-29

5. Cook, R.L., Torrance, K.E.: A Reflection Model for Computer Graphics. ACM Transactions on Graphics, Vol. 1, No.1 (1982) 7-24

6. He, X.D., Torrance, K.E., Sillion, F.X., Greenberg, D.P.: A Comprehensive Physical Model for Light Reflection. In Thomas, J.J.(ed.): Computer Graphics (Proceedings of ACM SIGGRAPH '91 Conference), Vol. 25. Annual Conference Series, ACM Press, New York (1991) 175-186 
7. Neyret, F., Heiss, R. Senegas, F.: Realistic Rendering of an Organ Surface in Real-Time for Laparoscopic Surgery Simulation. The Visual Computer. Vol.18, No.3 (2002) 135-149

8. Perlin, K., Hoffert, E.: Hypertexture. In Thomas, J.J.(ed.): Computer Graphics (Proceedings of ACM SIGGRAPH '89 Conference), Vol. 22. Annual Conference Series, ACM Press, New York (1989) 253 - 262

9. David, S.E. (ed.): Texturing and Modeling: A Procedural Approach. Morgan Kaufmann (2002)

10. Prusinkiewicz, P. Lindenmayer, A.: The Algorithmic Beauty of Plants. Springer-Verlag, New York (1990)

11. Weber, J., Joseph, P.: Creation and rendering of realistic trees. In Mair, S.G., Cook, R. (eds.): Computer Graphics (Proceedings of ACM SIGGRAPH '95 Conference). Annual Conference Series, ACM Press, New York (1995) 119-128

12. Perlin, K.: An Image Synthesizer. In Cole, P., Heilman, R., Barsky, B.A. (eds.): Computer Graphics (Proceedings of ACM SIGGRAPH '85 Conference), Vol.19, No.3. Annual Conference Series, ACM Press, New York (1985) 287-296

13. Fernando, R., Kilgard, M.J.: The Cg Tutorial. Addison Wesley (2003)

14. Chung, A.J., Deligianni, F., Shah, P., Wells, A., Yang, G.Z.: Enhancement of Visual Realism with BRDF for Patient Specific Bronchoscopy Simulation. In Barillot, C., Haynor, D.R., Hellier, P. (eds.): Lecture Notes in Computer Science, Vol. 3217. SpringerVerlag, Berlin Heidelberg New York (2004) 486 - 493

15. Mark, W.R., Glanville, R.S., Akeley K., Kilgard, M.J.: Cg: A System for Programming Graphics Hardware in a C-Like Language. ACM Transactions on Graphics, Vol. 22, No.3 (2003) 896-907 